\title{
OXIMETRIA DE PULSO NA AVALIAÇÃO DO TRANSPORTE DE OXIGÊNIO EM PACIENTES CRÍTICOS
}

\author{
Wilma Aparecida Nunes* \\ Renato Giuseppe Giovanni Terzi**
}

NUNES, W.A.; TERZI, R.G.G. Oximetria de pulso na avaliação do transporte de oxigênio em pacientes críticos.

Rev.latino-am.enfermagem, Ribeirão Preto, v. 7, n. 2, p. 79-85, abril 1999.

Neste estudo a monitorização de 35 pacientes de terapia intensiva, submetidos a ventilação mecânica, permitiu estabelecer o nível de precisão de dois oxímetros de pulso de diferentes fabricantes. O desempenho foi determinado em função de dados comparativos obtidos pela hemo-oximetria, para proceder a análise estatística foi utilizado o teste t. Pacientes críticos são portadores de condições clínicas que podem, eventualmente, limitar o uso desta técnica. No presente trabalho foi demonstrado que a discrepância nos resultados obtidos pode ser atribuída a presença de metemoglobina e possivelmente à anemia associada a hipóxia.

UNITERMOS: oximetria, pulso, pacientes críticos, monitorização

\section{INTRODUÇÃO}

A Unidade de Terapia Intensiva reúne uma população com doenças agudas reversíveis que teria pouca possibilidade de sobrevivência em outros setores do hospital. Nessas condições, o que se busca em primeira instância é garantir níveis adequados de oxigênio no sangue arterial para evitar a hipóxia tecidual (EDWARDS, 1991).

A avaliação de transporte de oxigênio é possível pela mensuração de algumas variáveis e pelo cálculo de outras. Para tanto são instituídos procedimentos invasivos, que se por um lado são úteis, pois permitem avaliar o consumo e suprimento de oxigênio, por outro exigem manipulação invasiva, que aumenta o risco de agravamento do estado geral do paciente (TULLI et al., 1990).

O oxímetro de pulso permite uma monitorização contínua e não invasiva da saturação parcial de oxigênio $\left(\mathrm{psO}_{2}\right)$, que expressa a relação entre oxiemoglobina $\left(\mathrm{cO}_{2} \mathrm{Hb}\right)$ e a soma das concentrações de oxi e deoxiemoglobina $(\mathrm{cHb})$. O termo parcial é utilizado porque somente uma porção do total de hemoglobina é considerada, ou seja, aquela disponível para o transporte de oxigênio, podendo ser referenciada como saturação funcional (ZANDER \& MERTZLUFFT, 1990). A saturação parcial de oxigênio é expressa pela seguinte equação:

$$
\mathrm{psO}_{2}=\frac{\mathrm{cO}_{2} \mathrm{Hb}}{\left(\mathrm{cO}_{2} \mathrm{Hb}+\mathrm{cHb}\right)}
$$

$\mathrm{cO}_{2} \mathrm{Hb}=$ concentração de oxiemoglobina $\mathrm{cHb}=$ concentração de deoxiemoglobina

A monitorização de variáveis fisiológicas é essencial para conduzir o tratamento de pacientes críticos (GOLDEHEIM \& KAZEMI, 1984), porém a validade da informação depende da técnica executada para adquirila, da exatidão dos dados fornecidos e do quanto irá repercutir sobre o resultado da assistência prestada. Os membros da equipe de saúde envolvidos diretamente na assistência do paciente crítico, estão preocupados com a utilização indiscriminada dos equipamentos, e questionam a influência deles, no tratamento e resultado obtidos (HANNING \& ALEXANDER, 1995).

Dispor de método não invasivo capaz de estimar a oxigenação do sangue é uma perspectiva atraente para profissionais da terapia intensiva, sendo assim o presente estudo teve por objetivo:

1. Verificar a confiabilidade de medidas da saturação

* Enfermeira, Professora do Curso de Especialização em U.T.I. da Pontifícia Universidade Católica de Campinas, Supervisora do Núcleo de Assistência Médica Intensiva do Hospital de Clínicas - UNICAMP e Doutoranda da Escola de Enfermagem da Universidade de São Paulo

** Professor Adjunto do Departamento de Cirurgia da Faculdade de Ciências Médicas - UNICAMP 
arterial de oxigênio em pacientes internados na Unidade de Terapia Intensiva e submetidos a assistência respiratória mecânica, utilizando a oximetria de pulso. 2. Avaliar o desempenho de dois oxímetros de pulso, de diferentes fabricantes e estabelecer o grau de exatidão e precisão tendo como padrão a hemo-oximetria.

\section{METODOLOGIA}

O presente estudo foi realizado no Núcleo de Assistência Médica Intensiva do Hospital de Clínicas da Universidade Estadual de Campinas (HC-UNICAMP). A população foi constituída de 35 pacientes submetidos a assistência respiratória mecânica, nos meses de janeiro e fevereiro de 1993. Foram incluídos os pacientes que necessitavam de exames de gasometria, a pedido do médico intensivista, para acompanhamento clínico.

Os dados foram obtidos de acordo com o formulário elaborado (Anexo 1) para este fim, contendo variáveis, cujos valores poderiam contribuir para atingir os objetivos propostos. Os pacientes previamente selecionados, seguindo os critérios de inclusão, foram monitorizados com os dois oxímetros de pulso simultaneamente.

O oxímetro de pulso fornece leituras da saturação do sangue, avaliando o comportamento de absorção da oxiemoglobina e deoxiemoglobina em relação aos comprimentos de luz vermelha e infravermelha. $\mathrm{O}$ aparelho possui um receptáculo para acomodar a porção distal do dedo, com um dos lados contendo uma fonte de luz - composta de dois fotoemissores de luz (LED) - e do outro lado um fotodetector. Um LED emite luz vermelha ( $\cong 660 \mathrm{~nm})$ e outro luz infravermelha $(\cong 940 \mathrm{~nm})$ (Fig. 1) (ALEXANDER et al., 1989).

O oxímetro é equipado com um sistema dedicado (composto pelo software e hardware), e o alinhamento dos componentes permite assumir que toda luz incidente sobre o fotodetector tem o comprimento de onda do LED iluminante. Em alguns modelos a leitura é processada em três intervalos de tempo: um para o comprimento de onda vermelha, um para a infravermelha e outro com os dois desligados. Este último intervalo de tempo é utilizado para captar a luz ambiente, possibilitando a compensação do efeito dessa interferência sobre o fotodetector (TREMPER \& BARKER, 1987).
Para fornecer uma medida contínua da oxigenação arterial analisando a luz que atinge o fotodetector, o oxímetro de pulso possui dois componentes. O primeiro é o componente basal (CB), com uma transmitância constante ao longo do tempo, originária do conjunto de elementos não pulsáteis, formados por tecidos, capilares, sangue venoso e pele. $\mathrm{O}$ segundo componente é pulsátil (CP), decorrente do fluxo fásico de sangue arterial no leito tecidual, que muda de intensidade no tempo, em sincronia com o ciclo cardíaco. Durante a sístole há um aumento do volume de sangue o que promove maior absorção da luz, com decréscimo correspondente na transmitância; durante a diástole, quando diminui o volume sangüíneo, haverá aumento proporcional na intensidade da luz transmitida (Fig. 1) (TREMPER \& BARKER, 1987).



\section{Figura 1 - Descrição gráfica de fatores que interferem na absorção da luz}

A mudança de absorção da luz em função da pulsação é utilizada para o cálculo da $\mathrm{psO}_{2}$ baseada na razão (R) de transmitância da luz absorvida nos dois comprimentos de onda. Nos oxímetros de pulso somente a absorbância do componente pulsátil entre a fonte e o fotodetector é considerada decorrente do sangue arterial. A absorbância dos dois componentes ( $\mathrm{CB}$ e $\mathrm{CP}$ ) nos dois comprimentos de onda estabelece uma razão empiricamente correlacionada com a saturação (Fig. 2). O cálculo desta razão é uma forma de estabelecer uma correção, para aplicar-se a lei de Lambert-Beer*** em situações clínicas, nas quais não há possibilidade de mensuração de todas variáveis determinantes da medida.

\footnotetext{
*** Existe uma relação entre a concetração de um soluto e a luz transmitida através de uma solução $\mathrm{L}$ trans $=\mathrm{x} \mathrm{e}-\mathrm{A}$ onde $\mathrm{A}=\mathrm{D}$ $\mathrm{x} C \mathrm{x}$ e

$\mathrm{L}$ trans $=$ intensidade da luz transmitida

$\mathrm{L}$ in = intensidade da luz incidente

$\mathrm{A}=$ absorção

$\mathrm{D}=$ distância percorrida pela luz na solução

$\mathrm{C}=$ concentração do soluto
} 


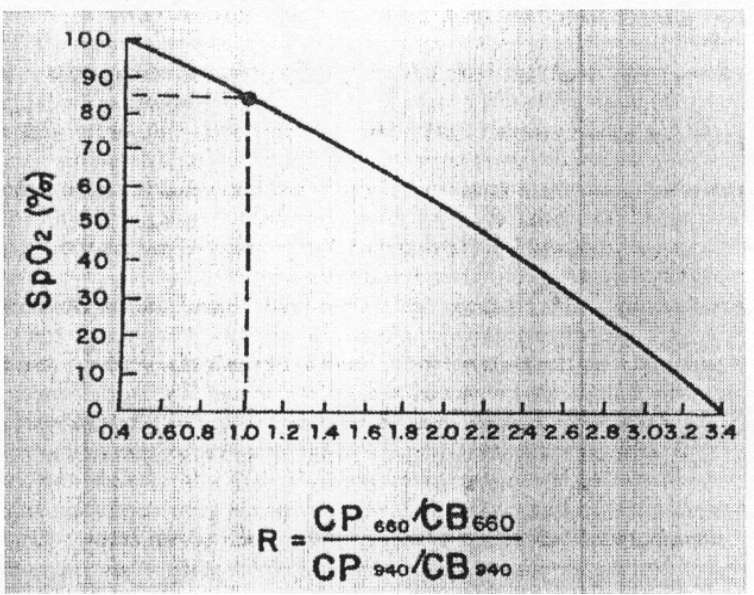

Figura 2 - Curva de calibração do oxímetro de pulso, baseada em dados experimentais obtidos por Pologe. Adaptado de TREMPER \& BARKER, 1987.

Os equipamentos eram: dois oxímetros de pulso portáteis capazes de monitorizar a saturação da hemoglobina arterial e o pulso, baseados nos princípios da espectrofotometria e plestimografia de diferentes fabricantes, o Pulsat ${ }^{\mathrm{IM}}$ (Spectramed inc. CCD) e o Satlite TRANS $^{\mathrm{TM}}$ (DATEX/Intrumentarium Corp); e um hemooxímetro capaz de medir automaticamente o total de hemoglobina, de oxiemoglobina, de carboxiemoglobina, de metemoglobina e conteúdo de oxigênio numa amostra de sangue total, o IL-282 ${ }^{11}$ (Instrumental Laboratory). Os resultados encontrados foram expressos em números absolutos e índices percentuais.

O sangue coletado em seringa de plástico previamente heparinizada com $0,2 \mathrm{ml}$ de heparina de uma solução de 5000 unidades por $\mathrm{ml}$ de anticoagulante e lubrificada com movimentos do êmbolo. Ao término da coleta a seringa era vedada e procediam-se movimentos rotatórios para homogeneizar a amostra; os exames do material foram realizados em laboratório dentro da unidade.

\section{RESULTADOS E DISCUSSÃO}

O diagnóstico em si pode ser insuficiente para estabelecer a gravidade dos pacientes. KNAUS et al. (1981) demonstraram que aproximadamente metade dos pacientes admitidos em terapia intensiva está no período pós-operatório de cirurgias eletivas, com baixo risco de mortalidade e necessitando exclusivamente de monitorização. Neste trabalho eles são 9 e perfazem $25,75 \%$ da população estudada.

Observa-se no Quadro 1, que os níveis de carboxiemoglobina determinados pela hemo-oximetria estiveram dentro do intervalo de normalidade - inferior a $2 \%$ em humanos, e portanto não poderiam afetar a fidedignidade dos valores de saturação fornecidos pelo oxímetro de pulso. Os níveis aumentam em caso de tabagistas crônicos ou em situações de inalação de fumaça, a leitura elevada ocorre porque, no comprimento de onda $660 \mathrm{~nm}$, a carboxiemoglobina tem um coeficiente de extinção de 0,07 , que é praticamente indistinguível do coeficiente da oxiemoglobina neste comprimento de onda, associado ao fato do coeficiente de extinção da carboxiemoglobina ser zero a $940 \mathrm{~nm}$. Assim sendo aumento na concentração de carboxiemoglobina tende a diminuir a razão com a qual a saturação está correlacionda (EISENKRAFT, 1988).

A metemoglobina aparece em nível extremamente elevado no décimo paciente de nossa casuística, submetido a tratamento com nitrato substância que facilita o acúmulo de metemoglobina por ser metabolizada no fígado para gliceril dinitrato e nitrito. O nitrito favorece a conversão da $\left(\mathrm{Hbfe}^{++}\right) \mathrm{em}\left(\mathrm{Hbfe}^{+++}\right)$, reduzindo a capacidade de transporte de oxigênio (KUMAR et al., 1990). Quando a percentagem de metemoglobina aumenta, a razão de absorção 660/940 tende para a unidade e a leitura da $\mathrm{psO}_{2}$ aproxima-se de $85 \%$, independente do percentual de oxi e deoxiemoglobina presentes no sangue. Haverá um falso aumento da $\mathrm{psO}_{2}$ com baixa saturação arterial e os valores estarão subestimados em situações de alta saturação arterial (DELWOOD et al., 1991). Como se tratava de um paciente ictério portador de insuficiência hepática e renal, fazendo uso de nitrato endovenoso, foi possível detectar níveis de metemoglobina de $18 \%$ e de oxiemoglobina de $81,3 \%$. Por outro lado, observa-se que os dois oxímetros de pulso forneceram um valor de 99 para as leituras de saturação neste paciente, quando a leitura esperada com a elevação da metemoglobina seria 85\%. BEALL \& MOORTHY (1989), demonstraram que os níveis séricos de metemoglobina podem não ser compatíveis com as leituras elevadas fornecidas pelo hemo-oxímetro, em pacientes com hiperbilirrubinemia. Nesta circunstância, segundo eles, os resultados da oximetria de pulso estarão mais fidedignos do que aqueles obtidos pela hemo-oxímetria. Com base nos resultados desses estudos, podemos pressupor que os níveis de metemoglobina determinados pela hemo-oxímetria poderia não ser da magnitude registrada. Este fenômeno adquire importância na medida que o óxido nítrico, vem sendo cada vez mais utilizado na Unidade de Terapia Intensiva, no suporte ventilatório de pacientes com Síndrome da Angústia Respiratória do Adulto (S.A.R.A.). 
Quadro 1 - Descrição dos valores obtidos na análise direta da amostra de sangue dos pacientes estudados $\left(\mathrm{O}_{2} \mathrm{Hb}\right.$, MetHb, COHb, $\left.\mathrm{pO}_{2}, \mathrm{pCO}_{2}, \mathrm{Hb}\right)$ e calculado $\left(\mathrm{caO}_{2}\right)$ UTI-HC-UNICAMP, 1993

\begin{tabular}{|c|c|c|c|c|c|c|c|}
\hline $\mathbf{N}^{\mathbf{o}}$ & $\% \mathrm{O}_{2} \mathrm{Hb}$ & \%MetHb & $\%$ СОНb & $\mathrm{PO}_{2}(\mathrm{mmHg})$ & $\mathrm{pCO}_{2}(\mathrm{mmHg})$ & $\mathrm{Hb}(\mathrm{g} / \mathrm{dl})$ & $\mathrm{caO}_{2}$ \\
\hline 1 & 97.50 & 0.70 & 0.70 & 160.60 & 30.20 & 9.10 & 12.33 \\
\hline 2 & 98.70 & 0.00 & 0.80 & 271.60 & 56.10 & 11.80 & 16.19 \\
\hline 3 & 97.10 & 1.20 & 1.00 & 143.80 & 24.40 & 8.20 & 11.07 \\
\hline 4 & 96.60 & 1.50 & 0.70 & 82.80 & 28.70 & 8.00 & 10.74 \\
\hline 5 & 97.70 & 0.30 & 0.30 & 106.70 & 42.40 & 10.20 & 13.85 \\
\hline 6 & 97.20 & 0.00 & 2.40 & 139.20 & 39.00 & 12.10 & 16.35 \\
\hline 7 & 99.10 & 0.50 & 1.20 & 375.00 & 31.00 & 12.30 & 16.95 \\
\hline 8 & 97.60 & 0.10 & 0.70 & 93.20 & 30.60 & 14.70 & 19.94 \\
\hline 9 & 97.50 & 0.70 & 0.70 & 166.30 & 32.60 & 9.80 & 13.28 \\
\hline 10 & 81.30 & 18.00 & 0.00 & 159.00 & 28.00 & 16.00 & 18.08 \\
\hline 11 & 98.00 & 0.20 & 0.00 & 108.70 & 22.20 & 11.60 & 15.80 \\
\hline 12 & 97.20 & 0.00 & 0.70 & 136.10 & 24.20 & 9.40 & 12.70 \\
\hline 13 & 95.70 & 0.10 & 0.80 & 64.50 & 25.20 & 15.00 & 19.95 \\
\hline 14 & 96.90 & 0.30 & 1.00 & 76.60 & 22.60 & 11.20 & 15.08 \\
\hline 15 & 78.10 & 0.20 & 0.40 & 43.20 & 45.20 & 10.40 & 11.29 \\
\hline 16 & 84.20 & 0.00 & 0.90 & 50.30 & 34.30 & 14.30 & 16.73 \\
\hline 17 & 92.70 & 0.10 & 1.10 & 63.00 & 29.80 & 10.40 & 13.40 \\
\hline 18 & 96.50 & 0.50 & 0.90 & 80.60 & 31.50 & 11.80 & 15.83 \\
\hline 19 & 97.30 & 0.30 & 1.20 & 83.90 & 28.20 & 11.00 & 14.87 \\
\hline 20 & 98.40 & 0.40 & 1.80 & 168.00 & 27.50 & 14.70 & 20.11 \\
\hline 21 & 96.10 & 0.00 & 1.40 & 71.30 & 37.90 & 8.20 & 10.95 \\
\hline 22 & 98.00 & 0.20 & 1.50 & 153.10 & 37.70 & 11.80 & 16.07 \\
\hline 23 & 92.20 & 0.50 & 0.70 & 52.40 & 29.20 & 7.60 & 9.74 \\
\hline 24 & 98.60 & 0.50 & 1.10 & 128.30 & 23.20 & 10.80 & 14.80 \\
\hline 25 & 100.00 & 0.00 & 1.20 & 320.00 & 26.40 & 7.60 & 10.60 \\
\hline 26 & 98.50 & 0.30 & 0.50 & 132.50 & 33.70 & 9.70 & 13.28 \\
\hline 27 & 98.00 & 0.50 & 1.00 & 137.40 & 29.80 & 9.00 & 12.26 \\
\hline 28 & 98.40 & 0.50 & 1.10 & 141.60 & 36.30 & 13.20 & 18.05 \\
\hline 29 & 99.30 & 0.70 & 0.80 & 201.50 & 16.40 & 12.20 & 16.84 \\
\hline 30 & 98.30 & 0.20 & 0.70 & 124.80 & 26.10 & 7.90 & 10.79 \\
\hline 31 & 97.40 & 0.40 & 1.80 & 139.90 & 36.60 & 10.10 & 13.67 \\
\hline 32 & 98.30 & 0.10 & 0.70 & 103.70 & 25.00 & 10.90 & 14.89 \\
\hline 33 & 89.10 & 0.20 & 1.40 & 281.40 & 28.00 & 7.40 & 10.09 \\
\hline 34 & 98.60 & 0.00 & 0.50 & 190.00 & 33.00 & 13.30 & 18.23 \\
\hline 35 & 99.40 & 0.00 & 0.00 & 136.60 & 22.90 & 13.50 & 18.65 \\
\hline
\end{tabular}

$\% \mathrm{OHb}_{2}$ (percentagem de oxiemoglobina IL-282), \%MetHb (percentagem de metemoglobina IL-282), \%COHb (percentagem de carboxiemoglobina IL-282), $\mathrm{pO}_{2}$ (pressão parcial de oxigênio IL-813), $\mathrm{pCO}_{2}$ (pressão parcial de gás carbônico IL-813), $\mathrm{Hb}$ (concentração de hemoglobina), $\mathrm{caO}_{2}$ (conteúdo arterial de oxigênio) 
Quadro 2 - Apresentação dos valores de saturação obtidos por métodos invasivo e não invasivo UTI-HCUNICAMP, 1993

\begin{tabular}{|c|c|c|c|c|c|}
\hline $\mathbf{N}^{\mathbf{o}}$ & $\mathrm{SAT}_{1}$ & $\mathrm{SAT}_{2}$ & $\% \mathrm{O}_{2} \mathrm{Hb}$ & $\mathrm{DIF}_{1}$ & $\mathrm{DIF}_{2}$ \\
\hline 1 & 98 & 99 & 97.50 & 0.50 & 1.50 \\
\hline 2 & 99 & 99 & 98.70 & 0.30 & 0.30 \\
\hline 3 & 99 & 99 & 97.10 & 1.90 & 1.90 \\
\hline 4 & 96 & 95 & 96.60 & 0.60 & 1.60 \\
\hline 5 & 99 & 99 & 97.70 & 1.30 & 1.30 \\
\hline 6 & 94 & 99 & 97.20 & 3.20 & 1.80 \\
\hline 7 & 99 & 98 & 99.10 & 0.10 & 1.10 \\
\hline 8 & 99 & 99 & 97.60 & 1.40 & 1.40 \\
\hline 9 & 99 & 99 & 97.50 & 1.50 & 1.50 \\
\hline 10 & 99 & 99 & 81.30 & 17.70 & 17.70 \\
\hline 11 & 99 & 98 & 98.00 & 1.00 & 0.00 \\
\hline 12 & 97 & 98 & 97.20 & 0.20 & 0.80 \\
\hline 13 & 96 & 96 & 95.70 & 0.30 & 0.30 \\
\hline 14 & 99 & 96 & 96.90 & 2.10 & 0.90 \\
\hline 15 & 76 & 78 & 78.10 & 2.10 & 0.10 \\
\hline 16 & 83 & 83 & 84.20 & 1.20 & 1.20 \\
\hline 17 & 93 & 93 & 92.70 & 0.30 & 0.30 \\
\hline 18 & 99 & 98 & 96.50 & 2.50 & 1.50 \\
\hline 19 & 98 & 98 & 97.30 & 0.70 & 0.70 \\
\hline 20 & 98 & 99 & 98.40 & 0.40 & 0.60 \\
\hline 21 & 97 & 97 & 96.10 & 0.90 & 0.90 \\
\hline 22 & 99 & 99 & 98.00 & 1.00 & 1.00 \\
\hline 23 & 99 & 99 & 92.20 & 6.80 & 6.80 \\
\hline 24 & 99 & 99 & 98.60 & 0.40 & 0.40 \\
\hline 25 & 99 & 99 & 100.30 & 1.30 & 1.30 \\
\hline 26 & 99 & 99 & 98.50 & 0.50 & 0.50 \\
\hline 27 & 98 & 99 & 98.00 & 0.00 & 1.00 \\
\hline 28 & 98 & 99 & 98.40 & 0.40 & 0.60 \\
\hline 29 & 98 & 98 & 99.30 & 1.30 & 1.30 \\
\hline 30 & 98 & 99 & 98.30 & 0.30 & 0.70 \\
\hline 31 & 98 & 99 & 97.40 & 0.60 & 1.60 \\
\hline 32 & 98 & 98 & 98.30 & 0.30 & 0.30 \\
\hline 33 & 99 & 99 & 99.40 & 0.40 & 0.40 \\
\hline 34 & 97 & 99 & 98.60 & 1.60 & 0.40 \\
\hline 35 & 99 & 99 & 98.10 & 0.90 & 0.90 \\
\hline \multicolumn{6}{|c|}{$\begin{array}{l}\mathrm{SAT}_{1} \text { (saturação de oxímetro de pulso Satlite TRANS }{ }^{\mathrm{TM}} \text { ), } \\
\mathrm{SAT}_{2} \text { (saturação do oxímetro de pulso Pulsat }{ }^{\mathrm{IM}} \text { ), } \% \mathrm{O}_{2} \mathrm{Hb} \\
\text { (percentagem de oxiemoglobina do IL-282 = Padrão), DIF } \\
\text { (diferença em valor absoluto entre } \mathrm{SAT}_{1} \text { e o padrão), } \mathrm{DIF}_{2} \\
\text { (diferença em valor obsoluto entre } \mathrm{SAT}_{2} \text { e o padrão) }\end{array}$} \\
\hline
\end{tabular}

$\mathrm{Na}$ apresentação dos dados no Quadro 2 percebese que as diferenças entre o método invasivo e não invasivo estão acentuadas nos pacientes 10 (17.00) e 23 (6.80). Em nossa casuística observamos que a leitura no oxímetro de pulso não foi afetada em situações de anemia moderada $(\mathrm{Hb} \cong 8)$ na ausência de hipóxia. Quando a anemia foi moderada e associada à hipóxia, a queda nos valores de saturação acompanharam a queda nos níveis da $\mathrm{paO}_{2}$. Entretanto, observamos que a anemia associada à hipóxia provocou falhas na leitura, com o oxímetro de pulso registrando valores superestimados para a saturação.

Este fato aparentemente contraditório no paciente anêmico - cuja curva de dissociação da hemoglobina supõe-se desviada para a direita, de forma que a queda da pressão parcial de oxigênio no sangue estaria associada com queda na sua saturação - também foi observado por JAY \& RENZI (1992), que sugeriram dois mecanismos explicativos para a contradição: o primeiro, que a anemia não afeta significativamente o valor da absorção até que reduza a oxigenação da hemoglobina; e o segundo, que a eventual presença de hemoglobinas anômalas interferem nos resultados.

A surpreendente capacidade de tolerância do ser humano à hipóxia e a extensão do comprometimento orgânico determinado pela patologia de base são fatores que dificultam determinar o grau de hipoxemia tolerado, sem produzir sérios danos teciduais. Yelderman e New submeteram voluntários a hipóxia progressiva e estabeleceram que o oxímetro de pulso é preciso e exato no intervalo de saturação da hemoglobina de 50\%-100\% (YELDERMAN \& NEW, 1983).

Para proceder a análise estatística, inicialmente foi obtida a média, variância e desvio padrão para cada umas das variáveis, à exceção de duas variáveis distoantes, as distribuições de $\mathrm{DIF}_{1}$ e $\mathrm{DIF}_{2}$ se aproximam do normal. Essas variáveis correspondem aos pacientes 10 e 23 do Quadro 2 e são denominadas pontos fora ou "outliers". Os resultados são apresentados no quadro abaixo.

Quadro 3 - Descrição da média, variância e desvio padrão das variáveis UTI-HC-UNICAMP, 1993

\begin{tabular}{|lrrrrr|}
\hline & SAT $_{\mathbf{1}}$ & $\mathbf{S A T}_{\mathbf{2}}$ & $\mathbf{0}_{\mathbf{2}} \mathbf{H b}$ & DFF $_{\mathbf{1}}$ & DFF $_{\mathbf{2}}$ \\
\hline MÉDIA & 96.97 & 97.23 & 96.14 & 1.60 & 1.56 \\
VARIÂHCIA & 21.79 & 19.65 & 24.58 & 9.37 & 9.14 \\
DESVIO PADRÄO & 4.67 & 4.43 & 4.96 & 3.06 & 3.02 \\
\hline
\end{tabular}

Quadro 4 - Descrição da média, variância e desvio padrão com exclusão dos “outliers" das variáveis UTIHC-UNICAMP, 1993

\begin{tabular}{|lcrrrr|}
\hline & SAT $_{\mathbf{1}}$ & $\mathbf{S A T}_{\mathbf{2}}$ & $\mathbf{0}_{2} \mathbf{H b}$ & DFF $_{\mathbf{1}}$ & $\mathbf{D F F}_{\mathbf{2}}$ \\
\hline MÉDIA & 96.85 & 97.12 & 96.71 & 0.95 & 0.91 \\
WARIÂNCIA & 22.88 & 20.67 & 18.42 & 0.58 & 0.28 \\
DESVIO PADRÃO & 4.78 & 4.55 & 4.29 & 0.76 & 0.52 \\
\hline
\end{tabular}


Após exclusão dos valores "outliers" foi aplicado o teste de Kolmogorov para normalidade ao nível de significância $\propto=0.05$, rejeitou a hipótese de normalidade para $\mathrm{SAT}_{1} \mathrm{SAT}_{2} \% \mathrm{O}_{2} \mathrm{Hb}$, já para $\mathrm{DIF}_{1}$ e $\mathrm{DIF}_{2}$, o teste não rejeitou a hipótese de normalidade dos dados.

Considerando-se o fato de $\mathrm{DIF}_{1}$ e $\mathrm{DIF}_{2}$ representam o quanto o método não invasivo difere do método padrão foi aplicado o teste $t$, ao nível de significância $\propto=0.05$, não rejeitou a hipótese de igualdade das médias de $\mathrm{DIF}_{1}$ e $\mathrm{DIF}_{2}$, ou seja não existe diferença significativa (ao nível $\propto=0.05$ ) entre as médias de $\mathrm{DIF}_{1}$ e $\mathrm{DIF}_{2}$.

Foi feita uma análise a partir do intervalo de confiança para a razão das variância de $\mathrm{DIF}_{1}$ e $\mathrm{DIF}_{2}$. O intervalo de $95 \%$ de confiança obtido foi $(1.04 ; 4.27)$, significando que a variância de DIF é no mínimo igual, podendo chegar a quatro vezes a variância de $\mathrm{DIF}_{2}$.

\section{CONSIDERAÇÕES FINAIS}

A oximetria de pulso permite a monitorização não invasiva da saturação arterial em pacientes de terapia intensiva, submetidos à ventilação mecânica, porém não é um método adequado para todos os pacientes críticos.

Não há diferença significativa (ao nível $\propto=0.05$ ) entre os dois oxímetros de pulso estudados em relação ao hemo-oxímetro, ou seja ambos são exatos, no entanto, variabilidade nas leituras de saturação dos Satlite TRANS $^{\mathrm{TM}}$ é maior, o que confirma uma maior precisão do Pulsat ${ }^{\mathrm{TM}}$

\section{PULSE OXIMETERS IN THE EVALUATION OF OXYGEN TRANSPORTATION IN CRITICAL PATIENTS}

In this clinical investigation 35 patients under mechanical ventilation were studied. It was possible to establish the precision of two pulse oximeters of different brands. The performance of these equipments was evaluated by comparing data with the hemo-oximeter and a statistical analysis employed the student $t$ test. Results showed that bias between oximeters reading and hemo-oximeter was similar for both instruments. Eventually critical patients may present conditions that limit the use of this technique. The study showed that the discrepancy in the results observed may be attributed to the presence of the methemoglobin and possible to anaemia associated to hipoxia.

\section{KEY WORDS: oxymetry, pulse, critical patients, monitoring}

\section{OXIMETRÍA DE PULSO EN LA EVALUACIÓN DEL TRANSPORTE DE OXÍGENO EN PACIENTES CRÍTICOS}

En este estudio el monitoraron 35 pacientes de cuidado intensivo, submetidos a ventilación mecánica, permitiendo establecer el nivel de precisión de 2(dos) oxímetros de pulso de diferentes fabricantes. El desempeño fue determinado en función de datos comparativos obtenidos por la hemo-oximetría. Para proceder al análisis estadístico, fue utilizado el ensayo t. pacientes críticos son portadores de condiciones clínicas que pueden, eventualmente, limitar el uso de esta técnica. En el presente trabajo, fue demostrado que la discrepancia en los resultados obtenidos, puede ser atribuida a la presencia de metemoglobina e posiblemente a la anemia asociada a la hipoxia.

TÉRMINOS CLAVES: oximetría, pulso, pacientes críticos, monitoraron

\section{ANEXO 1}

\begin{tabular}{ll}
\hline NOME & $\mathrm{N}^{\mathrm{o}}$ de UTI \\
\hline SEXO & IDADE
\end{tabular}

DIAGNÓSTICO PRINCIPAL

\begin{tabular}{llll}
\hline PULSAT $^{\mathrm{IM}}$ & \multicolumn{2}{c}{ SATLITE TRANS } \\
\hline SAT & PULSO & SAT & PULSO \\
& & & \\
\hline TEMP & FREQ RESP & $\mathrm{FiO}_{2}$ & PAS/PAD
\end{tabular}

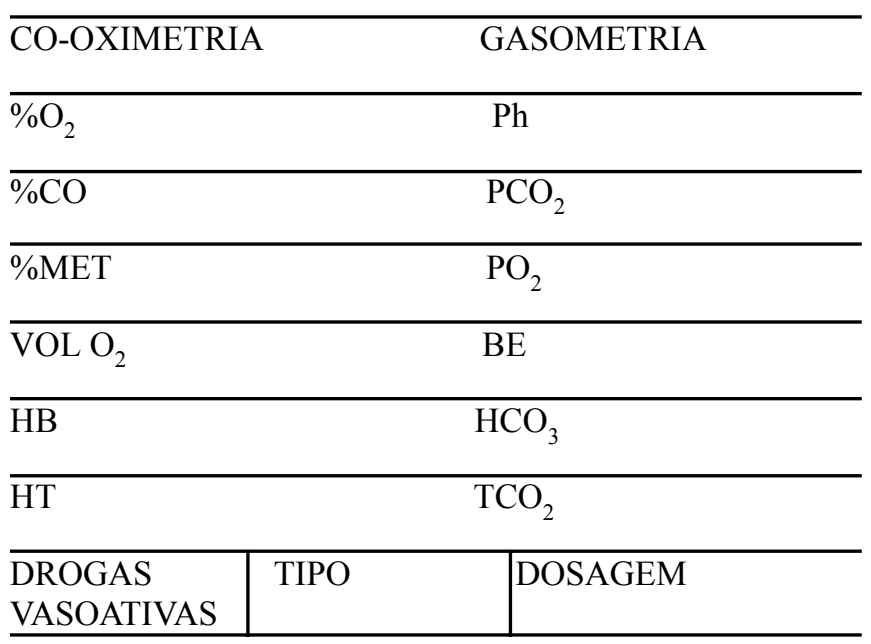




\section{REFERÊNCIAS BIBLIOGRÁFICAS}

01. ALEXANDER, C.M.; TELLER, L.E.; GROSS, J.B. Principles of pulse oximetry: theoretical and pratical considerations. Anaesth.Analg., v. 68, n. 3, p. 368-76, 1989

02. BEALL, S.N.; MOORTHY, S.S. Jaundice, oximetry and spurius hemoglobin desaturation. Anaesth.Analg., v. 68, n. 3, p. 806-7, 1989.

03. DELWOOD, L. et al. Methemoglobinemia and its effects on pulse oximetry. Crit.Care Med., v. 19, n. 7, p. 988, 1991.

04. EDWARDS, J.D. Oxygen transport in the critically III. Intens.Crit.Care Digest, v. 10, n. 2, p. 23-5, 1991.

05. EISENKRAFT, J.B. Carboxyhemoglobin and pulse oximetry. Anesthesiology, v. 68, n. 2, p. 279-82, 1988.

06.GOLDENHEIM, P. D.; KAZEMI, H. Cardiopulmonary monitoring of critically ill patients. (part 1). N.Engl.J.Med., v. 311, n. 11, p. 717-21, 1984.
07. HANNING, C.D.; ALEXANDER, W. Pulse oximetry: a pratical review. BMJ, v. 311, p. 36770, 1995.

08. JAY, G.D.; RENZI, F.P. Evaluation of pulse oximetry in anemia from hemoglobin-H disease. Ann.Emerg.Med., v. 21, n. 5, p. 572-4, 1992.

09. KNAUS,W.A.; WAGNER, D.P.; DRAPER, E.A. The range of intensive care services today. JAMA, v. 246, n. 23, p. 2711-6, 1981.

10. KUMAR, A. et al. Nitrobenzene poisoning and spurious pulse oximetry. Anesthesiology, v. 45, p. 949-51, 1990.

11. TREMPER, K.K.; BARKER, S.J. Pulse oximetry. Anesthesiology, v. 70, p. 98-108, 1987.

12. TULLI, G. et al. The oxygen status of the arterial blood in the critically ill. Scand.J.Lab.Invest., v. 50, p. 107-18, 1990. Suppl. 203.

13. YELDERMAN, M.; NEW, W. Evaluation of pulse oximetry. Anesthesiology, v. 62, p. 85-7, 1983.

14. ZANDER, R.; MERTZLUFFT, F. Oxygen parameters of blood: definitions and symbols. Scand.J.Lab.Invest., v. 50, p. 187-96, 1990. Suppl. 203. 